\title{
Towards Shrewd Object Visualization Mechanism
}

\begin{abstract}
ISSN: 2641-3086
DOI: https://dx.doi.org/10.17352/tcsit

Received: 31 August, 2020

Accepted: 28 November, 2020

Published: 30 November, 2020

*Corresponding author: Shahzad Ashraf, College of Internet of Things Engineering, Hohai University Changzhou, Jiangsu, China, E-mail:nfc.iet@hotmail.com

Keywords: Network forensics; Human computer interaction; Cyber warfare
\end{abstract}

\author{
Shahzad Ashraf* \\ College of Internet of Things Engineering, Hohai University Changzhou, Jiangsu, China
}

https://www.peertechz.com

Check for updates

\section{Abstract}

In order to measure the accurate outcome of different visualization mechanism it is imperative to adopt a shrewd strategy. Indeed, the outcome of experiment is focusing to assess the value of complex visualization approaches when comparing with alternative methods for data analysis. The interaction between participant prior knowledge and experience, a diverse range of experimental or real -world data sets and a dynamic interaction with the display system presents challenges when seeking timely, affordable and statistically relevant results. A hybrid approach proposed is being proposed to deal with complex interactive data analysis tools. This approach involves a structured survey completed after free engagement with the software platform by expert participants. The survey captures objective and subjective data points relating to the experience with the goal of making an assessment of the software performance supported by statistically significant experimental results. This work is particularly applicable to field of network analysis for cyber security and also military cyber operations and intelligence data analysis.

\section{Introduction}

Visualization is the key bridge between the data's quantitative content and human experience, and it can be argued that there is nothing (including mathematical constructs) that we cannot imagine in any way that we can truly understand or intuitively understand. Human beings possess an excellent pattern-recovery mechanism in our brains, which is critically dependent on our ability to conduct efficient and scalable visual discovery in data-based research. This may be one of the most critical methodological problems in 21st century for data-rich research [1].

With the passage of time, the $3 \mathrm{D}$ visualisation technology is demystifying rapidly and it becomes crucial utilizing the Augmented Reality (AR), Virtual Reality (VR) and high-quality displays for generation [2], of abstract and highly interactive tools for data analysis. There exists some extensive and established phenomenon emphasizing how to avail the Virtual Reality (VR) and Immersive Virtual Reality (IVR) [3]. Due to high cost and lack of portability, limited processing capacity and bespoke supporting software frameworks have hindered broader application. Due to a continue chain upgradation in sensor technology mainly used for Electronic Warfare (EW), Intelligence and Cyber Warfare has led to massive stores of data, which requires some assisted process to generate actionable knowledge [4].
Virtual reality has been shown in areas where the key dimensions are spatial to contribute to better exploration. It has shown that data visualization facilitates possessed highly abstract multidimensional analyses [5]. Many researchers are looking to display large datasets and fusion of interactive virtual and abstract visualization and the interplay between new technologies and human perception are one of the aims we strive to explore. In order to enhance scientific results, VR and abstract data visualization have both been independently demonstrated, but to the best of our knowledge, there is no assessment of how interactive virtual reality can use abstract representation of high-dimensional data to promote scientific research [6]. Since immersive visualization will improve the productivity of desktop visualization, we believe that immersive visualization is one of the foundations for exploring the greater dimensionality and abstraction of "big data". Some crucial cases are the studies being cited, they appear to be very casespecific (or one-of) and we know of no VR method that has tried to leverage the visual and interactive problem-solving of abstract, multi-dimensional data for the general purpose [7]. In other words, the use of interactive VR for data exploration as a general-purpose tool. Traditional methods require the use of very complex, costly and non-portable facilities. Our objective is to develop efficient, scalable, portable data visualization software available with low prices, commercially developed hardware on a standard affordable desktop or laptop. 
The first priority is an efficient visualization of highly dimensional data in some high dimensional space of data parameters that can be expressed as feature vectors (or data points). We intend to imagine in the future non-discrete, continuum or multidimensional density fields.

In certain cases, the statistically important experiment is triggered by interactive and immersive visualizations [8]. In terms of user interaction, visualization typically is highly dynamic and the synthesis of expertise by an analyst depends heavily on an established understanding of the underlying field. How can one or one component of a visualization be better calculated as a different approach, considering these challenges?.

Our findings suggest an alternative approach to the efficiency of an interactive visualization, particularly for applications where human-based laboratory experiments are less successful or where participants are difficult to locate. This research is focused on the engagement of experts with a visualization tool, accompanied by the completion of a survey providing choices for subjective and objective answer. An overview of the proposed method, anticipated effects, and how the fields in which the approach can be successful would follow this. In conclusion, it will discuss suggested potential work [9].

Experimentation varies from assessment within software engineering and related methods. An assessment will show that a piece of software is capable of achieving the objectives of a set of use cases but tests are typically focused on comparison and performed under controlled circumstances with the intent of drawing a statistically significant conclusion. A recent example of a software experiment, when applied to AR and VR, is the Tilt Map [10]. A group of 12 participants in this study participated in a 3D immersive environment with three different types of data presentation. From this for a well-defined mission, it was possible to produce a statistically significant response to questions about speed and precision.

In certain cases, where the study purpose is to compare interaction with a data set in an immersive analysis method with an established approach to analysis that might focused on textural or $2 \mathrm{D}$ graphical analysis [11]. It is more difficult to design experiments that are feasible within a realistic number of participants and time resources. Research tasks that cannot be easily divided into simple, discrete tasks as the synthesis of information from contact with data is harder to perform experiments.

The issue emerged from this a small, well established workflow experimental understanding cannot translate into an understanding or change for larger, related tasks. Experimental findings can also not extend to conditions in the real world. It is fair to assume that the experiment would offer an appreciation in the method if a company is to invest heavily in improvements to its processes. However other experimental alternatives are appropriate for circumstances where the interaction is too difficult to capture in controlled experiments.

Furthermore, the use of experiments has another constraint because it is difficult to collect appropriate participants in certain areas. For example, there is a shortage of employees in the niche knowledge area of network analysis for cyber security. A statistically meaningful sample of laboratory-based test results is an unparalleled job. With VR and AR applied to more niche and accurate skills, this problem will develop.

\section{Literature review}

Owing to the lack of a clear approach for the evaluation of information presentation methods for various types of displays, there have been experiences of contradictory findings going through a large literature review. The cyber security with an immersive data visualisation tool has been used by Kullman, et al. [12]. This research was presented as a usability analysis between $2 \mathrm{D}$ and $3 \mathrm{D}$ representations of data. This approach involved exposing analysts to $2 \mathrm{D}$ data representations of a network before exposing them to a VR based representation of the same network data and then interviewing them about the experience.

The majority of experimentation examples provided within the HCI Experimentation focus on sub-components of an interface. For example, comparing node graph layouts for readability and comparing web page layouts for ascetic value [13]. When the proposed tool is a combination of several methods of data presentation, there is a level of interaction with the data during the course of analysis and an analysis goal which may not be easily defined. In this case it is difficult to design an experiment with sufficient granularity to assess the overall performance of the software tool.

An efficient approach becomes imperative that can be classed as experimentation rather than evaluation. Experiments are characterized as comparative, formal and quantitative, whereas evaluation is non-comparative, informal and subjective. In some scenarios, where the goal is to choose between candidate solutions for data visualisation as part of a business process there is need for the level of confidence provided by experimentation [14], however, there is also a need to deal with the challenge of limitations on participant numbers, physical access to testing facilities and the duration of experimentation. The use of immersive spellbound opens the field of multisensory displays of information utilizing a sense of touch and proprioception, a perception of body position knowledge and movement to intuitively and attractively experience data i.e. with meaningful experiences and input from the force. The author [15], also reported that immersion into the presentation of knowledge improves information decision-making and encourages social involvement in the collaborative environment. Immersive visualisations allow to move into the data and interact with data using your whole body via multisensory input and output devices. Devices such as TUIs [16], allow for the contact of gestures and various parts of the body, encourage this.

\section{key challenges to suggested approaches}

It is important to have an experimental method to assess complex data analysis software, significant effort has been expended in building infrastructure to support this type of testing. The examples discussed previously focus on discrete 
components of the visualisation, with a view to generalising results to the whole process of analysis. The challenges with this approach include, measuring and accounting for the knowledge and experience of the participants, collecting experimental data during highly complex interaction with the data, difficulty in identifying quantitative measures during exploratory tasks that can be generalized [17].

In an existing, live system, the interaction between the prior knowledge and experience of a subject matter expert, their individual approach to information discovery and the way they will interact with a complex visualisation is very difficult to account for in an achievable experiment. Testing of participants prior to the experiment, or collection of domain experience information can be useful where the group size is large, and the diversity of skill levels is distinct enough to create sub-groups. In most cases this is not possible in the participant numbers available for HCI experimentation.

It is possible to collect quantitative data around every physical interaction a participant has with a visualisation system, be it 2-dimensional, 3-dimensional, VR, or AR. There are multiple ways to achieve this, including tracking eye movement collection and analysis of mouse and keyboard inputs, and the use of verbal 'thinking out loud' recordings to gain insight into the thought process being used by the participant. With this data availability, it is difficult to draw conclusion around the connection between these metrics and knowledge obtained by the analyst. Where an analyst is zooming, rotating, re-positioning and filtering aspects of a complex visualizations, and where there are many millions of different chains of interaction that might lead to the same discovery, it would be impossible to measure the contribution that individual elements of the visualisation made to the discovery of knowledge. Even if discrete activities were designed in the experiment to focus on elements such as colour selection, node layout modes, zoom levels, filtering methods, it would still be difficult to argue how much each aspect contributes to the knowledge discovery process in the full system [18].

Considering these issues in the field of network security information a comparative evaluation was conducted by [19], with 8 students involved training on two separate tools and the conduct of well-defined comparison and identification tasks followed by an exploratory activity. This activity was also focused on the analysis of computer network traffic. Goodall highlights the challenges in trying to collect quantitative measures of activities which involve discovery of unknown information within a data set. One of the purported benefits of immersive data analysis is the hope of generating knowledge from the data that was previously unavailable when using automated sorting and categorisation, 2D graphing, artificial intelligence or rules-based data analysis. An experimental approach which involves intentionally hiding knowledge and then seeing whether a group of analysts can find it, is only valid for the specific information that was hidden. This approach does not allow for the discovery of previously unidentified knowledge during the experiment. For the said reasons, an approach where participants interact with a visualisation tool using real data of their own is proposed as a new method for experimentation in the field of interactive data analysis [20].

The 11 study participants are listed in Table 1. Participants from the industry include both data analysts and managers/ executives. At the respective companies, interviews and seminars were held.

\section{Proposed approach}

Finding suitable and sufficient number of participants is remained a challenging task attending and controlling the lab-based, experiments for interactive software experiments. When specific software is being targeted at a specific subset of participants, such as network security analysts, rather than the general population, there are additional geographical and availability challenges. In this proposed approach, participants are asked to interact with the spellbound approach under assessment and then complete a survey consisting of qualitative and quantitative questions as illustrated in Figure 1. In addition, information regarding their level of experience in the specific analysis field and their experiences with existing analysis approaches is collected in the same survey response.

It is the authors assertion that the concept of abstracting interactions away from real world scenarios down to simplistic separations or identification of colours, shapes or arbitrary artificial data weakens the ability to apply the experimental outcomes to specific real-world areas of data analysis. Breaking the analysis process into slices for the purpose of making inperson experimentation achievable also weakens the ability to

Table 1: During the interview and workshop, analysis approach and study participants.

\begin{tabular}{|c|c|c|c|}
\hline \multirow{2}{*}{$\begin{array}{c}\text { Research } \\
\text { Approach }\end{array}$} & \multicolumn{2}{|c|}{ Role of the participants } & \multirow{2}{*}{ Nature of the organization } \\
\cline { 2 - 4 } & Data analyst & $\begin{array}{c}\text { Data } \\
\text { caretaker }\end{array}$ & \\
\hline Interview session & 1 & 1 & $\begin{array}{c}\text { Related to business and } \\
\text { management }\end{array}$ \\
\hline In practice & - & 1 & $\begin{array}{c}\text { About accounting and auditing } \\
\text { Morkshop }\end{array}$ \\
\hline
\end{tabular}

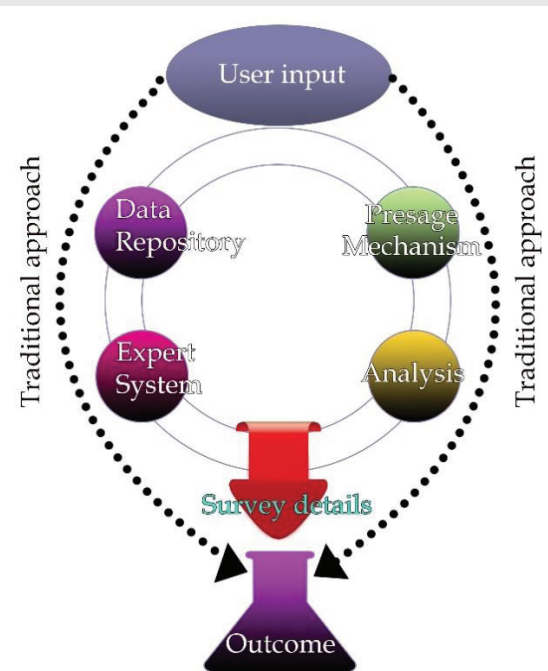

Figure 1: The proposed Model. 
extrapolate to the full analysis process. Allowing analysts to follow the full analysis process with real-world data makes it much easier to use this data in support of performance assessment, and potentially allows for a more cohesive response.

The proposed experimental approach for this study was developed to use in the assessment of a specific $3 \mathrm{D}$ analysis tool for computer network traffic in support of cyber security operations. However, the approach could be applicable in any case where a data discovery task is to be carried out on data which is multi-variate and nodal in some dimensions. The approach seeks to avoid the complexities and challenges introduced by in-lab experimentation while still generating a statistically significant result. Due to the unique nature of discovering knowledge or insights from complex data, the ideal state is to have analysts interact with a real-world data set that they are familiar with. Mostly likely they will have already used other tools to interact with this data and have an idea of what they already expect to discover. In this approach, the visualisation assessment is provided to the participants using for own computers, with their own data set. A minimum time for interaction should be set, possibly 1 hour, and a maximum limit for 2-3 hours. Participants can then be given an online survey which combines scaled and free text questions about the system under test, the existing approaches used to conduct analysis and relevant background information.

The approach which would be used by a participant in normal circumstance, the Existing Analysis Tools, do not need to be disclosed to the researcher, only a response in the survey regarding the performance of the tools and a broad categorisation is required. Using the results of the anonymous web-based survey, an experimental assessment of the propose visualisation can then be generated. This approach avoids requiring access by the researchers to possibly sensitive data or analysis methodologies already in use by the participants.

\section{The disadvantages}

An experimental approach that relies on unsupervised interaction with software does have several risks and possible disadvantages when compared with structured, directly observed experimentation in a controlled environment. Some of the expected risks and possible mitigations are discussed below.

\section{Technical aspects}

With participants using their own data and their own computer hardware, there is a risk that challenges getting the software to work will result in failure to effectively participate. There is also the issue that performance, especially for immersive $3 \mathrm{D}$ visualisations, will be a factor in the way that participants interact with their data. This may be mitigated by indicating a minimum hardware specification and providing detailed installation and use instruction videos. There is also the possibility of incorporating a performance self-test within the distributed software to ensure a minimum threshold of performance.

\section{Dataset variations}

Allowing participants to analyse data of their own interest, removes the ability of researchers to control this variable. Traditionally, tight control of the experimental objects is at the heart of experimental software assessment. Of course, it is possible to ship a common set of test data with the software, however, this removes the benefit of familiarity and motivation. Participating analysts are likely to spend more time and be more motivated to work with data that they already have an interest in and more likely to discover new information in a dataset that they are already familiar with. Participants are also more likely to easily complete the survey questions relating to their Existing Analysis Tools when working on data they have already analyzed. The intended mitigation for this problem is to include in the survey sufficient detail to describe the test data, without breaching any privacy or security concerns.

\section{Cumulative response}

Using an anonymous web-based survey carries the risk of responses that have been completed with intentionally inaccurate or random responses. This is a common risk to all experimental approaches that rely on responses from participants that cannot be physically or logically verified. Figures 2,3 illustrates the 3D network spellbound mechanism. It is not possible to know if a participant claims 5 new insights when using the visualisation under assessment when in fact they had none. A mitigation to this risk will be including qualitative free-text responses to be written in support of quantitative responses.

\section{Performance and results}

The experimentation approach proposed in this paper has been developed for the assessment of the Scanmap3D [21] visualisation tool for network traffic analysis. The application of user-based experimentation is proposed as a complementary activity to the procedural based testing. The Scanmap3D tool presents network traffic captures as a 2D layout of nodes, representing network hosts, with a vertical stack of glyphs representing packs. Analysists are able to shuffle the $2 \mathrm{D}$ layout and rotate, pan, zoom and filter the display. The goal of experimentation is to compare the performance of this tool against existing analysis approaches. Responses to survey

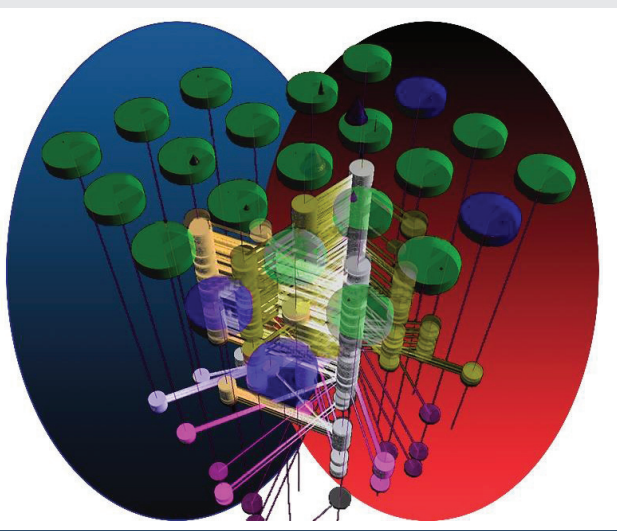

Figure 2: Network Visualisation appears in 3D illustration. 


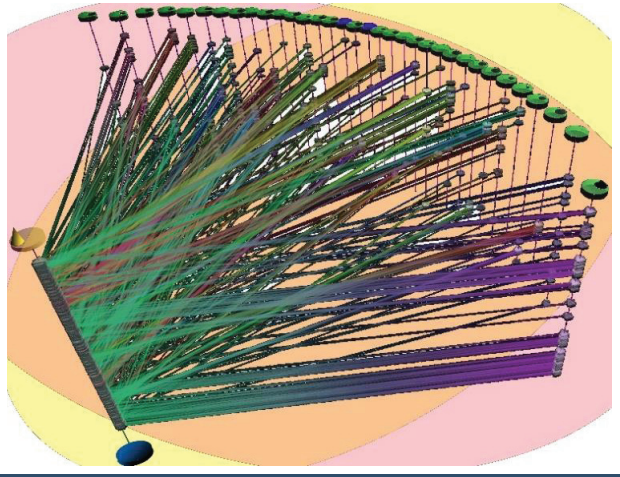

Figure 3: Network spellbound mechanism.

requests based on utilisation of the software tool will be used to generate an assessment of the performance of the software compared with existing methods for network traffic analysis. By limiting the participant group to those analysts already working in the field of network traffic analysis, the expectation is that the toolset already in use will be representative of the industry in general. Survey questions which generate quantitative responses around the process and performance of the software will be combined with comparative process and performance responses for how the participant would complete the same discovery task with existing tools. It is expected that broad description of the existing preferred approach, such as $2 \mathrm{D}$ graphs or database queries, will allow for statistical grouping of the individual participant responses with the purpose of comparing the assessed visualisation against specific types of analysis, rather than just against all other analysis methods.

In addition to the assessment of performance and process, the survey will also be utilised to collect metrics around the preference, perception [6] aspects of the participant experience to assist with further development of the software. A small subset of the expected data is being captured using the participant surveys. It will be possible to graph against other collected metrics such as level of experience of analyst, and also grouping of existing tools into common types.

\section{Application to broader experimentation}

While proposed approach is assessing a new data visualisation and analysis tool against existing approaches could be used in any situation where a visual, interactive abstraction of data is used for the purpose of knowledge discovery [22]. In cases where the task being assessed is one of classification or sorting, it is likely that more traditional laboratory-based testing against experimental objects that closely reflect the intended use case would be a better option. However, in cases where a complex set of interactions by the analyst effectively generates different experimental objects with each iteration, this approach, focused on the knowledge discovered rather than the process, may be applicable.

There are also cases where this experimental approach could be combined with other assessment approaches such as procedural analysis [23], of the discovery steps, or modelling of the analyst process. This assessment approach might be unique during periods where in-person experimentation is not possible, such as under the COVID-19 pandemic. The proposed results have been illustrated through Figures 4,5.

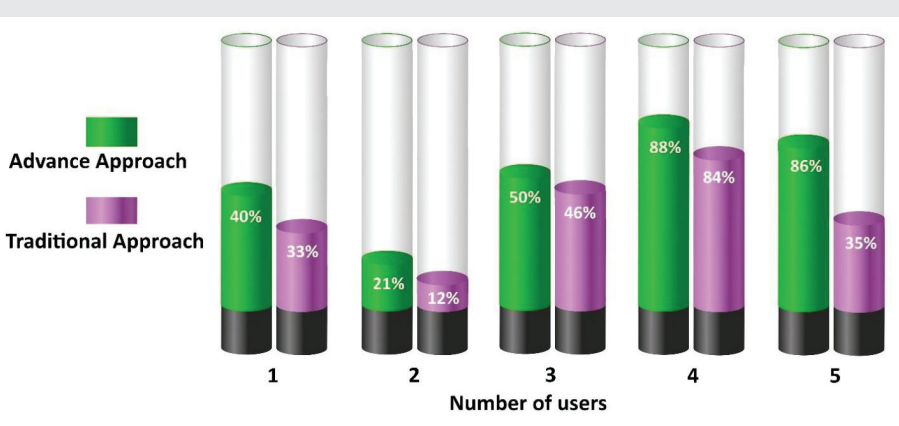

Figure 4: The traditional approach.

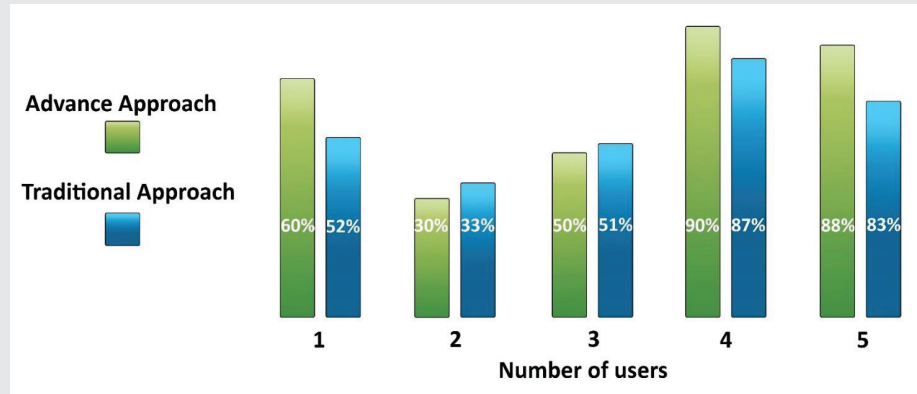

Figure 5: The spellbound approach.

\section{Conclusion and future work}

This paper outlines an alternate process for evaluating the effectiveness of immersive visualisations, specifically in VR and AR environments. This approach takes a broader, more systemic perspective than many of the accepted processes in this field. It is currently being trailed in experiments in the field of computer and network security, although it is the authors assertion that this framework is designed to be applicable to a broader community. The results of this experimentation will be combined with procedural assessment of the Scanmap3D software using an expert analyst to provide an overall assessment of the effectiveness of this approach to immersive visualisation of analysis of network traffic.

The resulting survey responses from participants in this experiment could provide valuable insights into how best to create survey questions that elicit useful responses for assessment of immersive data analysis software.

The results of the experimentation conducted for this research will have direct implication for the field of data analytics in a military context. The fields of Cyber warfare, Intelligence and Electronic Warfare are increasingly faced with large amounts of nodal data which is inherently suited to visual analysis approaches and it is critical to have a robust process for measuring performance of candidate technologies.

\section{References}

1. Samaniego-Franco JB, Jara-Roa DI, Sarango-Lapo CP, Agila-Palacios 
MV, Guaman-Jaramillo JE, et al. (2018) Case study: Methodology for the development of learning objects $(\mathrm{OA})$ in $3 \mathrm{D}$ for applications of augmented reality (AR). 1-7. Link: https://bit.ly/2V9o6v4

2. Plunkett KN (2019) A Simple and Practical Method for Incorporating Augmented Reality into the Classroom and Laboratory. J Chem Educ 96: 26282631. Link: https://bit.ly/3nVBJuq

3. Shahzad A, Tauqeer A (2020) Dual-nature biometric recognition epitome Trends Comput Sci Inf Technol 5: 008-014. Link: https://bit.ly/3liRGce

4. Ashraf S, Saleem S, Chohan AH, Aslam Z, Raza A (2020) Challenging strategic trends in green supply chain management. J Res Eng Appl Sci 05: 71-74. Link:

5. Purchase HC (2012) Experimental Human-Computer Interaction: A Practical Guide with Visual Examples. Illustrated edition. Cambridge: Cambridge University Press. Link: https://bit.ly/3o7czJt

6. Ashraf S, Muhammad D, Aslam Z (2020) Analyzing challenging aspects of IPv6 over IPv4. J Ilm Tek Elektro Komput Dan Inform 6: 54. Link: https://bit.ly/3mfB7zl

7. Easterbrook S, Singer J, Storey MA, Damian D (2008) Selecting Empirical Methods for Software Engineering Research. In Guide to Advanced Empirical Software Engineering F. Shull, J. Singer, and D. I. K. Sjøberg, Eds. London: Springer 285-311. Link: https://bit.ly/3q3Tee1

8. Saleem S, Ashraf S, Basit MK (2020) CMBA - A Candid Multi-Purpose Biometric Approach. ICTACT J Image Video Process 11. Link: https://bit.ly/39uq9SO

9. Ashraf S, Saleem S, Ahmed T, Aslam Z, Muhammad D (2020) Conversion of adverse data corpus to shrewd output using sampling metrics. Vis Comput Ind Biomed Art 3. Link: https://bit.ly/2VdxA8R

10. Ashraf S (2019) Culminate Coverage for Sensor Network through BodaciousInstance Mechanism. Manag J Wirel Commun Netw 8.

11. Ashraf S, Ahmed T, Raza A, Naeem H (2020) Design of Shrewd Underwater Routing Synergy Using Porous Energy Shells. Smart Cities 3: 74-92. Link: https://bit.ly/2JqclOA

12. Kullman K, Asher NB, Sample C (2019) Operator Impressions of 3D Visualizations for Cybersecurity Analysts. Link: https://bit.ly/36gfZmM https://bit.ly/3fH2yQ2

13. Ashraf S, Muhammad D, Shuaeeb M, Aslam Z (2020) Development of Shrewd Cosmetology Model Through Fuzzy Logic. J Res Eng Appl Sci 5: 93-99. Link: https://bit.ly/3q9VrEw

14. Ashraf S, Ahmed T, Saleem S, Aslam Z (2020) Diverging Mysterious in Green Supply Chain Management. Orient J Comput Sci Technol 13: 22-28. Link: https://bit.ly/2V9oQjQ

15. Ashraf S, Gao M, Chen Z, Kamran S, Raza Z (2017) Efficient Node Monitoring Mechanism in WSN using Contikimac Protocol. Int J Adv Comput Sci Appl 8. Link: https://bit.ly/3o2D2HV

16. Ashraf S, Saleem S, Afnan S (2020) FTMCP: Fuzzy based Test Metrics for Cosmetology Paradigm. Adv Comput Intell Int J ACII 4: 1-13. Link: https://bit.ly/33oe6CG

17. Ashraf S, Muhammad D, Khan MA, Ahmed T (2020) Fuzzy based efficient Cosmetology Paradigm. 8: 513-520.

18. Ashraf S, Saleem S, Ahmed T, Aslam Z, Shuaeeb M (2020) Iris and Foot based Sustainable Biometric Identification Approach. in International Conference on Software, Telecommunications and Computer Networks (SoftCOM), Split, Hvar, Croatia.

19. Goodall JR (2009) Visualization is better! A comparative evaluation. In 2009 6th International Workshop on Visualization for Cyber Security 57-68. Link: https://bit.ly/2HL31Ep

20. Ashraf S, Ahmed T (2020) Machine Learning Shrewd Approach Fo An Imbalanced Dataset Conversion Samples. J Eng Technol 11. Link: https://bit.ly/33nbGEH

21. Ashraf S, Aslam Z, Saleem S, Afnan S, Aamer M (2020) Multi-biometric Sustainable Approach for Human Appellative. CRPASE Trans Electr Electron Comput Eng 6: 146-152. Link: https://bit.ly/3nZViBR

22. Ashraf S, Ahmed T, Saleem S (2020) NRSM: node redeployment shrewd mechanism for wireless sensor network. Iran J Comput Sci. Link: https://bit.ly/2HOAv4O

23. Ashraf S, Saleem S, Ahmed T (2020) Sagacious Communication Link Selection Mechanism for Underwater Wireless Sensors Network. Int J Wirel Microw Technol 10: 22-33. Link: https://bit.ly/2J7yd1r

\section{Discover a bigger Impact and Visibility of your article publication with} Peertechz Publications

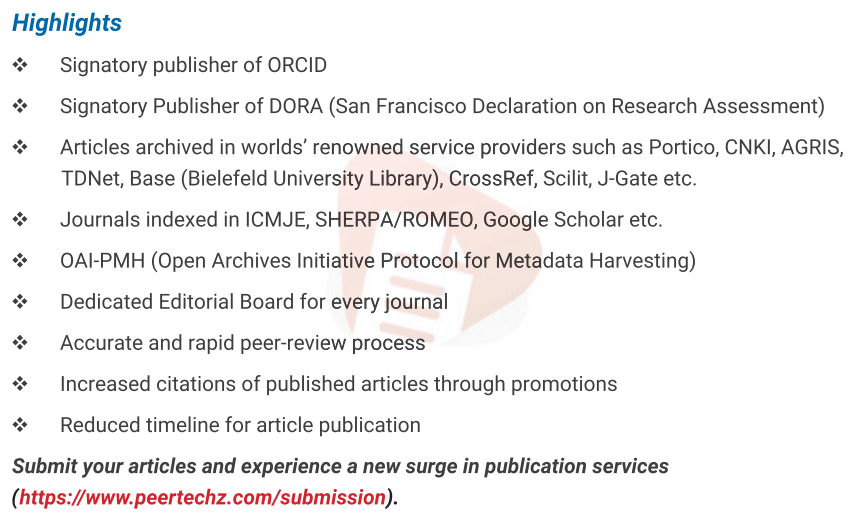

Peertechz journals wishes everlasting success in your every endeavours.

Copyright: (c) 2020 Ashraf S. This is an open-access article distributed under the terms of the Creative Commons Attribution License, which permits unrestricted use, distribution, and reproduction in any medium, provided the original author and source are credited. 